\title{
Evaluation of the impact of delayed centrifugation on the diagnostic performance of serum creatinine as a baseline measure of renal function before antiretroviral treatment
}

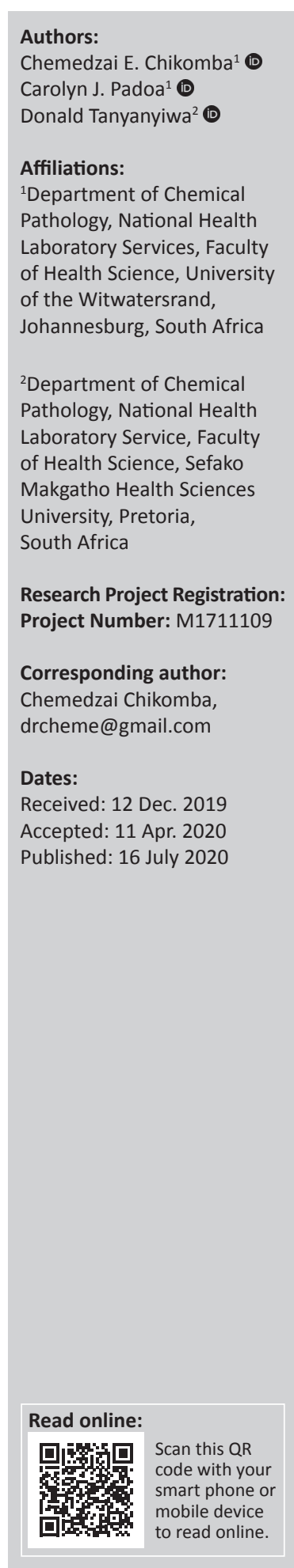

\begin{abstract}
Background: The measurement of serum creatinine is a standard requirement of the medical management of people living with HIV. Renal dysfunction is common, both as a complication of HIV-infection and as a result of its treatment. The detection of abnormal renal function before the start of antiretroviral therapy will impact patient management and the outcome of treatment.

Objectives: This study aimed to determine if a time delay in the centrifugation of serum samples affected the creatinine level and the estimated glomerular filtration rate as recorded on the analytical platforms used in the laboratory.
\end{abstract}

Methods: Twenty-two $(n=22)$ HIV-positive, newly diagnosed and treatment-naïve patients were randomly recruited from Alexandra Health Clinic, Johannesburg, South Africa. Serum samples were centrifuged at six time intervals following receipt of the sample viz. $<4 \mathrm{~h}$ (baseline), $6 \mathrm{~h}, 24 \mathrm{~h}, 48 \mathrm{~h}, 72 \mathrm{~h}$ and $96 \mathrm{~h}$. Creatinine concentrations were measured on the Roche platform utilising the enzymatic and kinetic Jaffe methods. Whole blood samples were also analysed with the Abbott i-STAT point-of-care instrument. The estimated glomerular filtration rate was calculated using the Cockcroft Gault, CKD-Epidemiology Collaboration and Modified Diet and Renal Disease v3/4 equations.

Results: At baseline $(<4 \mathrm{~h})$ there was good agreement between the enzymatic and kinetic Jaffe methods: bias $1.7 \mu \mathrm{mol} / \mathrm{l}$. The enzymatic and i-STAT creatinine concentrations were stable over $96 \mathrm{~h}$ viz. changes of $1.8 \%$ and 5.7\%. However, from $24 \mathrm{~h}$ onwards agreement between the enzymatic and kinetic Jaffe methods was poor with the latter measuring $43.7 \mu \mathrm{mol} / 1$ higher than the enzymatic method at $96 \mathrm{~h}$. Creatinine concentrations measured with the kinetic Jaffe method increased significantly in samples centrifuged after $6 \mathrm{~h}(p<0.001,61.7 \%$ change), and resulted in a 95\% decline in eGFR at $96 \mathrm{~h}$ as determined with the CKD-Epidemiology Collaboration equation.

Conclusion: The analysis of serum creatinine using the isotope dilution mass spectrometry traceable kinetic Jaffe method is unreliable if performed on samples centrifuged $\geq 6 \mathrm{~h}$ after collection. The raised creatinine concentration can affect clinical decisions such as renal functional assessment, choice of antiretroviral drug or regimen, and the dose and frequency of medication.

Keywords: Kidney function; serum creatinine; antiretroviral; estimated GFR; kinetic Jaffe; i-STAT; ROCHE.

\section{Introduction}

South Africa (SA) has one of the highest HIV infection rates in the world. In 2016, the overall prevalence of HIV infection in SA was $12.7 \%{ }^{1,2}$ The use of antiretroviral drugs (ARVs) has slowed the progression of the infection and increased the life expectancy of people living with HIV., ${ }^{3,45}$ In SA's public health sector, patients are started on antiretroviral treatment (ART) with combinations of different ARV drug classes, prescribed as a single, once-a-day tablet. ${ }^{6}$ This has led to a reduction of the pill-burden of therapy and to improved adherence. ${ }^{7,8}$ In the southern African region the most frequently prescribed initial combination is the nucleotide reverse transcriptase

How to cite this article: Chikomba CE, Padoa CJ, Tanyanyiwa D. Evaluation of the impact of delayed centrifugation on the diagnostic performance of serum creatinine as a baseline measure of renal function before antiretroviral treatment. S Afr J HIV Med. 2020;21(1), a1056. https://doi.org/10.4102/sajhivmed.v21i1.1056

Copyright: ( 2020. The Authors. Licensee: AOSIS. This work is licensed under the Creative Commons Attribution License. 
inhibitor (NtRTI), tenofovir disoproxil fumarate (TDF), the nucleoside reverse transcriptase inhibitors (NRTIs) emtricitabine (FTC) or lamivudine (3TC) and the nonnucleoside reverse transcriptase inhibitor (NNRTI), efavirenz (EFV). This is first-line ART. ${ }^{6}$ Current SA national ART guidelines indicate that the integrase strand transfer inhibitor (INSTI), dolutegravir (DTG), has replaced efavirenz for all except women of child-bearing age who may fall pregnant or those initiating ART in the first trimester of pregnancy., ${ }^{9,10}$ Tenofovir however remains the standard NRTI-backbone of all first-line ART in southern Africa.

TDF is an acyclic nucleoside phosphate prodrug. Its long half-life permits once-daily dosing. Although the drug is extensively filtered by the kidneys, $20 \%-30 \%$ is actively reabsorbed at the proximal tubule. ${ }^{11}$ TDF is an occasional cause of renal injury viz. a proximal renal tubulopathy and a salt-wasting syndrome (Fanconi Syndrome and renal tubular acidosis), drug-induced acute kidney injury and a slower, general decline in glomerular filtration, diabetes insipidus, and a dysregulation of the kidney's calcium and phosphorus metabolism. ${ }^{11,12}$ Impaired renal function may also result from the use of other drugs, such as aminoglycosides, sulphonamides, and amphotericin B, the presence of HIVassociated nephropathy (HIVAN), blood-borne infection, for example tuberculosis, bacteraemia and fungaemia, HIVassociated immune-complex kidney disease and common comorbidities such as hypertension and diabetes mellitus. ${ }^{13,14}$ Pre-existing mild renal dysfunction may increase susceptibility to the toxicity of TDF. Kidney function must be evaluated before starting ART: the use of TDF is contraindicated if the estimated glomerular filtration rate (eGFR) is $<50 \mathrm{~mL} / \mathrm{min}$. Patients on TDF have serum creatinine measured at 3 and 6 months following the initiation of ART and biannually thereafter. In high-risk patients, for example with coexistent hypertension or diabetes, the creatinine is measured more frequently. ${ }^{9}$

The measurement of the GFR confirms and stages the degree of renal impairment and provides a platform for the ongoing monitoring of kidney function. Although the urinary clearance of inulin is the gold standard of GFR measurement, ${ }^{15}$ the method is time-consuming, expensive and impractical in most clinical settings. Endogenous substances such as serum creatinine and cystatin $C$ are cheaper, provide more rapid results and are widely available. ${ }^{16}$ Although the National Institute for Health and Care Excellence (NICE) and the Kidney Disease Improving Global Outcomes (KDIGO) groups both base their eGFR assessment (equations) on cystatin $C$, its cost and lack of standardisation excludes its general use. ${ }^{17,18}$ Creatinine is produced at a constant rate, is present in all body fluids, and is filtered by the glomerulus. Its serum level is influenced by several biological factors, such as active secretion by the renal tubules in the presence of declining renal function, diet, extremes of muscle mass, age, gender, drugs and the use of creatine supplements. ${ }^{19}$ Several equations control for some of these variables. ${ }^{20}$ The SA National Department of Health (SANDOH) 2019 guidelines recommend therefore that the Counahan-Barratt equation be used to measure the eGFR of youths aged 10-16 years and the Modifications of Diet in Renal Disease (MDRD) equation be used for adolescents and adults 16 years of age and older. ${ }^{9}$

The Cockcroft-Gault (CG) equation incorporates the serum creatinine level and the variables of weight, age and sex in the measurement of the eGFR. ${ }^{21}$ The equation has limitations: in pregnancy, at the extremes of weight, and in those on dialysis for acute renal failure. Its principal use is in drug dosing and pharmacokinetic studies. ${ }^{20}$ The MDRD equation adds a fourth variable: race. ${ }^{22}$ This ethnicity adjustment-factor is based on African Americans and tends to overestimate the GFR in sub-Saharan (African) populations. ${ }^{23}$ The CKD-Epidemiology Collaboration (EPI) equation is recommended by the KDIGO guidelines group and has been validated in participants with and without impaired renal function with eGFR $<90 \mathrm{~mL} /$ $\min / 1.73 \min ^{2} .^{17,24}$ The SA National Health Laboratory Service (SA-NHLS) currently reports both the MDRD and CKD-EPI eGFR without ethnic adjustment. The adoption of the CKD-EPI equation in SA has been delayed due to limited local data.

Routine laboratory serum creatinine measurements are analysed on Jaffe and enzymatic assays. These methods should be traceable to isotope dilution mass spectrometry (IDMS) method and the Standard Reference Material for creatinine in serum (SRM 967). NHLS laboratories in SA generally use the modified kinetic Jaffe method, as opposed to the enzymatic assay, as it is more affordable. The World Health Organization's (WHO) guidelines indicate that at room temperature, creatinine is stable for 2-3 days. ${ }^{25}$ However, a report from Shepherd et al. ${ }^{26}$ noted a significant increase in creatinine levels measured on the kinetic Jaffe method when processing occurred later than 24 h. ${ }^{26}$ This impacted the study's eGFR results and had caused the renal misclassification of patients. ${ }^{26}$ A turnaround time for serum creatinine measurements from local clinics in Gauteng of 12-72 $\mathrm{h}$ has been observed at NHLS laboratories, as reported in the internal TAT reports. This delay increases with the remoteness of clinics due to the poor transport networks, the restricted working hours at referral laboratories, and the greater workload of regional laboratories. The aim of this study was to determine, in HIV-positive black South Africans not on ART, the stability limit (SL), that is, the time at room temperature, when the serum creatinine results are still within the maximum permissible instability (MPI) range. ${ }^{27}$

\section{Methods Study population}

Twenty-two $(n=22)$ newly diagnosed and treatment-naïve people living with HIV between the ages of 18 and 70 years were randomly recruited from the Alexandra Health Community Centre in Alexandra, Johannesburg, SA. The minimum number of participants required for testing 
the stability of biochemical analytes is $10 .{ }^{27}$ Furthermore, multiple samples were required from each participant for analysis on three analysers at six time points, providing 396 creatinine measurement data points. Patients with comorbidities such as diabetes mellitus, pregnancy, urinary tract infection, hypertension and proteinuria were excluded from the study. De-identified demographic and anthropometric data were transcribed from the patient files.

\section{Sample collection and processing}

Each patient provided 13 tubes of blood: $12 \times$ approximately $2 \mathrm{~mL}$ in $5 \mathrm{~mL}$ serum separator-tubes (SST) and $1 \times 5 \mathrm{~mL}$ in a heparin tube (Becton Dickenson, Plymouth, UK). Serum was isolated from SST tubes following centrifugation at $1370 \times \mathrm{g}$ for $5 \mathrm{~min}$ at defined time intervals viz. $<4 \mathrm{~h}, 6 \mathrm{~h}, 24 \mathrm{~h}, 48 \mathrm{~h}$, $72 \mathrm{~h}$ and $96 \mathrm{~h}$. Prior to centrifugation, samples were stored at room temperature. Whole blood heparin tubes were stored at room temperature until required.

\section{Measurement of creatinine concentrations}

Serum creatinine concentrations were measured immediately after each centrifugation (as above) using the IDMS-traceable enzymatic and IDMS-traceable kinetic Jaffe method on the Roche COBAS 8000 module 702 and Roche COBAS 6000 module 502 instruments at the NHLS laboratory of the Chris Hani Baragwanath Hospital, Soweto, SA. The enzymatic method test principle is based on a series of coupled reactions that result in the conversion of creatinine to hydrogen peroxide. In the final reaction, catalysed by peroxidase, a quinone imine chromogen is formed. The colour intensity of this chromogen is measured at $550 \mathrm{~nm}$ and is directly proportional to the concentration of creatinine in the sample. The kinetic Jaffe method test principle is based on the reaction of creatinine and picrate under alkaline conditions forming a yellow-orange complex. The rate of formation of this complex is proportional to the amount of creatinine in the sample. The kinetic Jaffe method is prone to interference from noncreatinine chromogens such as proteins and ketones. A correction factor of $-26 \mu \mathrm{mol} / \mathrm{L}$ is, therefore, universally applied to correct for these inherent chromogens. Bilirubin is the major negative interferent in the kinetic Jaffe method, and this is overcome by rate blanking. The internal quality control for creatinine in our laboratory is performed twice daily. The laboratory adheres to the Royal College of Pathologists of Australasia (RCPA) quality assurance programmes. External quality assurance submissions during the study period were within allowable limits.

Creatinine concentrations were also measured using whole blood samples on the Abbott i-STAT point-of-care instrument immediately after collection and at 6 h, 24 h, 48 h, $72 \mathrm{~h}$ and $96 \mathrm{~h}$. This method is based on the conversion of creatinine to hydrogen peroxide, via hydrolysis and oxidation. The hydrogen peroxide is oxidized (at the platinum electrode) to produce a current that is proportional to the concentration of creatinine in the sample.

\section{Estimated glomerular filtration rate measurements}

eGFR was calculated using the MDRDv3 without ethnic adjustment (age, sex, and serum creatinine), MDRDv4 (age, sex, ethnicity, and serum creatinine), CG and CKD-EPI equations with ethnic adjustment.

\section{Statistical analysis}

All analyses were performed using Medcalc software. The Shapiro-Wilks test was used to test for normality. Normally distributed continuous variables (creatinine) were presented as mean \pm standard deviation and continuous variables that were not normally distributed (age, weight) were presented as median [interquartile range]. Categorical variables are presented as proportions and percentages. The Student paired t-test was used to compare baseline creatinine concentrations between the three different methods (Bonferroni corrected $p$-value). The serum creatinine results were evaluated for analytically significant changes using the uncertainty of measurement (UOM; calculated for the laboratory quality control data in the preceding 6 months), total allowable error (TAE) of 15\% (Clinical Laboratory Improvement Amendments [CLIA] and coefficient of variation $[\mathrm{CV}])$. Passing-Bablok linear regression and BlandAltman plots were generated to evaluate correlation and method agreement over time, using the enzymatic method as the reference method. For all analyses, $p<0.05$ was considered statistically significant.

\section{Ethical consideration}

Ethical approval was obtained from the University of the Witwatersrand Human Research Ethic Committee, reference number: M1711109. R14/49.

\section{Results \\ Characteristics of the study population}

A total of 22 randomly recruited HIV-positive, treatmentnaïve black South African individuals consented to participate in this study. The median age of the participants was 37 years. The majority $(54.5 \% ; 12$ of 22$)$ of participants were younger than 40 years of age. The age distribution is consistent with the current demographics published by Statistics South Africa with respect to individuals mostly affected by HIV/AIDS. All participants were black Africans (ethnicity self-reported). The median weight for the participants was $70.0 \mathrm{~kg}$ (range $45-110 \mathrm{~kg}$ ) and 59.1\% (13 of 22) of participants were male.

\section{Creatinine assay performance}

The mean baseline $(<4 \mathrm{~h})$ creatinine concentrations for the study cohort obtained using the enzymatic, kinetic Jaffe method and i-STAT methods were $78.73 \mu \mathrm{mol} / \mathrm{L}$ $\pm 14.63 \mu \mathrm{mol} / \mathrm{L}, 77.05 \mu \mathrm{mol} / \mathrm{L} \pm 13.12 \mu \mathrm{mol} / \mathrm{L}$ and 
$70.14 \mu \mathrm{mol} / \mathrm{L} \pm 15.3 \mu \mathrm{mol} / \mathrm{L}: p>0.05$ for all comparisons (Table 1). The mean creatinine concentrations analysed using the kinetic Jaffe method were significantly higher than baseline when blood samples were processed after $6 \mathrm{~h}$ : $p \leq 0.001$ for all comparisons. Creatinine concentrations did not change significantly over the $96 \mathrm{~h}$ when measured using the enzymatic method or i-STAT system: $p>0.05$ for all comparisons (Table 1).

The kinetic Jaffe method exhibited the widest intraindividual CV (range: $12 \%$ - $40 \%$ vs $1.2 \%$ - $12 \%$ enzymatic and $1.9 \%-12 \%$ i-STAT method) as well as the greatest interassay variation $(22.7 \%$ vs $6.0 \%$ enzymatic and $6.6 \%$ i-STAT method), as seen in Figure 1. When creatinine results were assessed against the $10 \% \mathrm{UOM}$ for each respective assay,
$8.9 \%$ of the enzymatic creatinine results fell outside the UOM for the duration of the study compared to $65 \%$ of the results for the kinetic Jaffe method. The i-STAT results could not be evaluated as UOM has not been established for this assay.

Based on the positive trend of increasing creatinine concentrations over time observed for the Jaffe method, we explored the data further to determine if a correction factor could be established to adjust creatinine results obtained from samples with delayed processing time. The percentage change of the serum creatinine results for the participants over the 96-h time frame was inconsistent and a correction factor could, therefore, not be established based on the current sample size (Figure 2).

TABLE 1: Creatinine concentrations for the study cohort $(n=22)$ obtained using the enzymatic, kinetic Jaffe method and i-STAT methods at six different time intervals.

\begin{tabular}{|c|c|c|c|c|c|c|}
\hline \multirow{3}{*}{$\begin{array}{l}\text { Time interval } \\
\text { (hours) }\end{array}$} & \multicolumn{6}{|c|}{ Creatinine concentration } \\
\hline & \multicolumn{2}{|c|}{ Enzymatic } & \multicolumn{2}{|c|}{ Kinetic Jaffe } & \multicolumn{2}{|c|}{ i-STAT } \\
\hline & $\mu \mathrm{mol} / \mathrm{I}$ & $* p$ & $\mu \mathrm{mol} / \mathrm{L}$ & $* p$ & $\mu \mathrm{mol} / \mathrm{L}$ & $* p$ \\
\hline$<4 h$ & $78.73 \pm 14.63$ & - & $77.05 \pm 13.12$ & - & $70.14 \pm 15.35$ & - \\
\hline $6 \mathrm{~h}$ & $74.55 \pm 14.11$ & 0.340 & $78.41 \pm 15.19$ & 0.752 & $69.91 \pm 16.00$ & 0.962 \\
\hline $24 \mathrm{~h}$ & $78.91 \pm 14.64$ & 0.967 & $95.23 \pm 20.26$ & 0.001 & $67.59 \pm 14.72$ & 0.578 \\
\hline $48 \mathrm{~h}$ & $79.86 \pm 15.70$ & 0.805 & $117.64 \pm 19.82$ & $<0.001$ & $68.18 \pm 14.02$ & 0.662 \\
\hline $72 \mathrm{~h}$ & $76.79 \pm 14.82 \dagger$ & 0.676 & $121.29 \pm 20.97 \S$ & $<0.001$ & $67.59 \pm 14.71$ & 0.577 \\
\hline $96 \mathrm{~h}$ & $77.31 \pm 10.45 \ddagger$ & 0.743 & $124.56 \pm 18.499$ & $<0.001$ & $66.14 \pm 15.82 \dagger \dagger$ & 0.406 \\
\hline
\end{tabular}

Results are expressed as mean \pm standard deviation, missing data due to insufficient volume or high haemolysis index (> $1000 \mathrm{mg} / \mathrm{dL}$ ) for

$\dagger, n=3$.

$\ddagger n=6$.

$\S, n=1$.

I, $n=4$.

$\dagger, n=1$.

$*, p$-value for results compared to $4 \mathrm{~h}$ creatinine concentration.

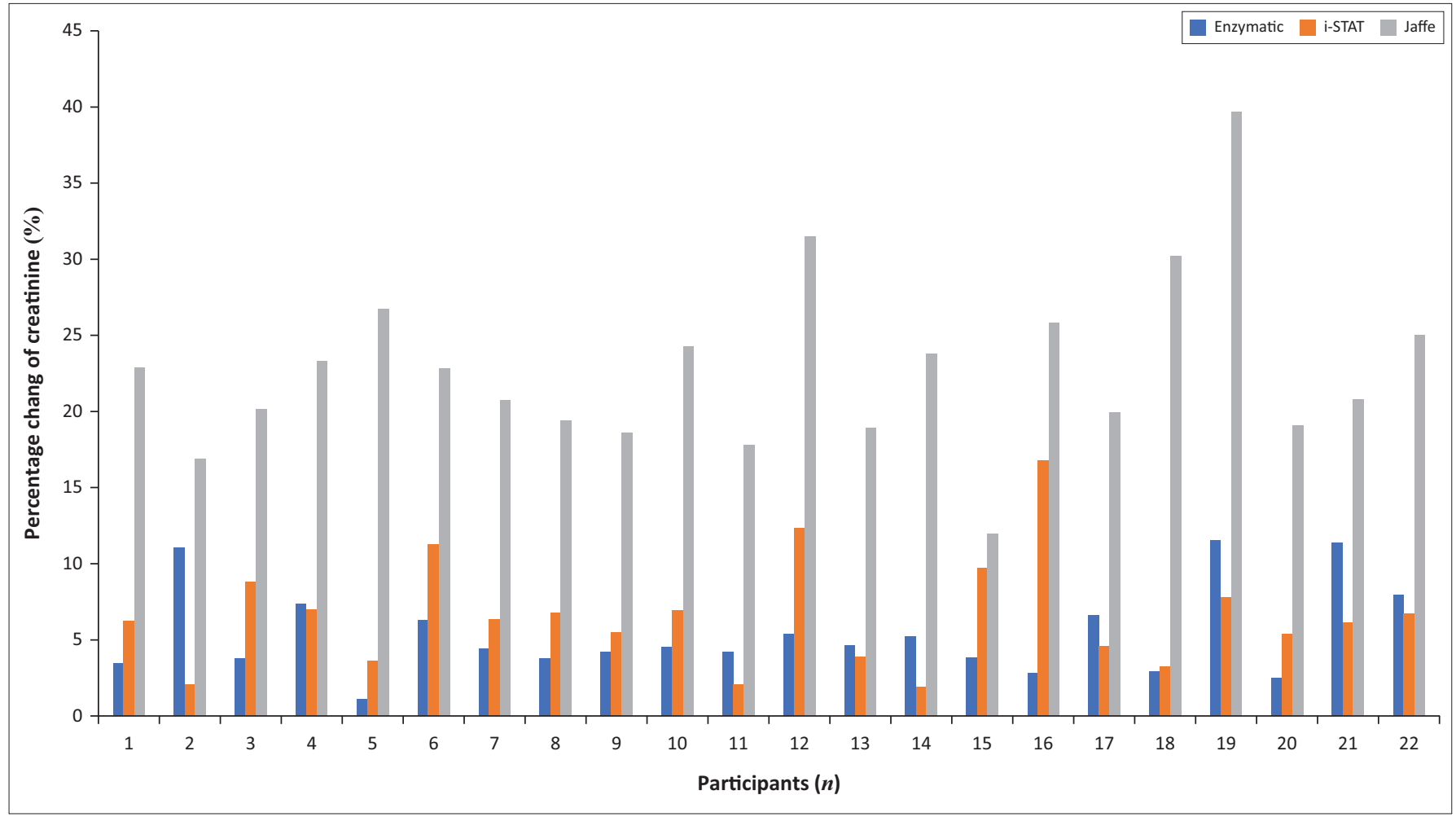

FIGURE 1: Intra-individual coefficient of variation for 22 participants for the enzymatic, kinetic Jaffe and i-STAT creatinine methods over the 96 -h study period. Blue bars represent results obtained for the enzymatic method, orange bars for the i-STAT method and grey bars for the kinetic Jaffe method. 


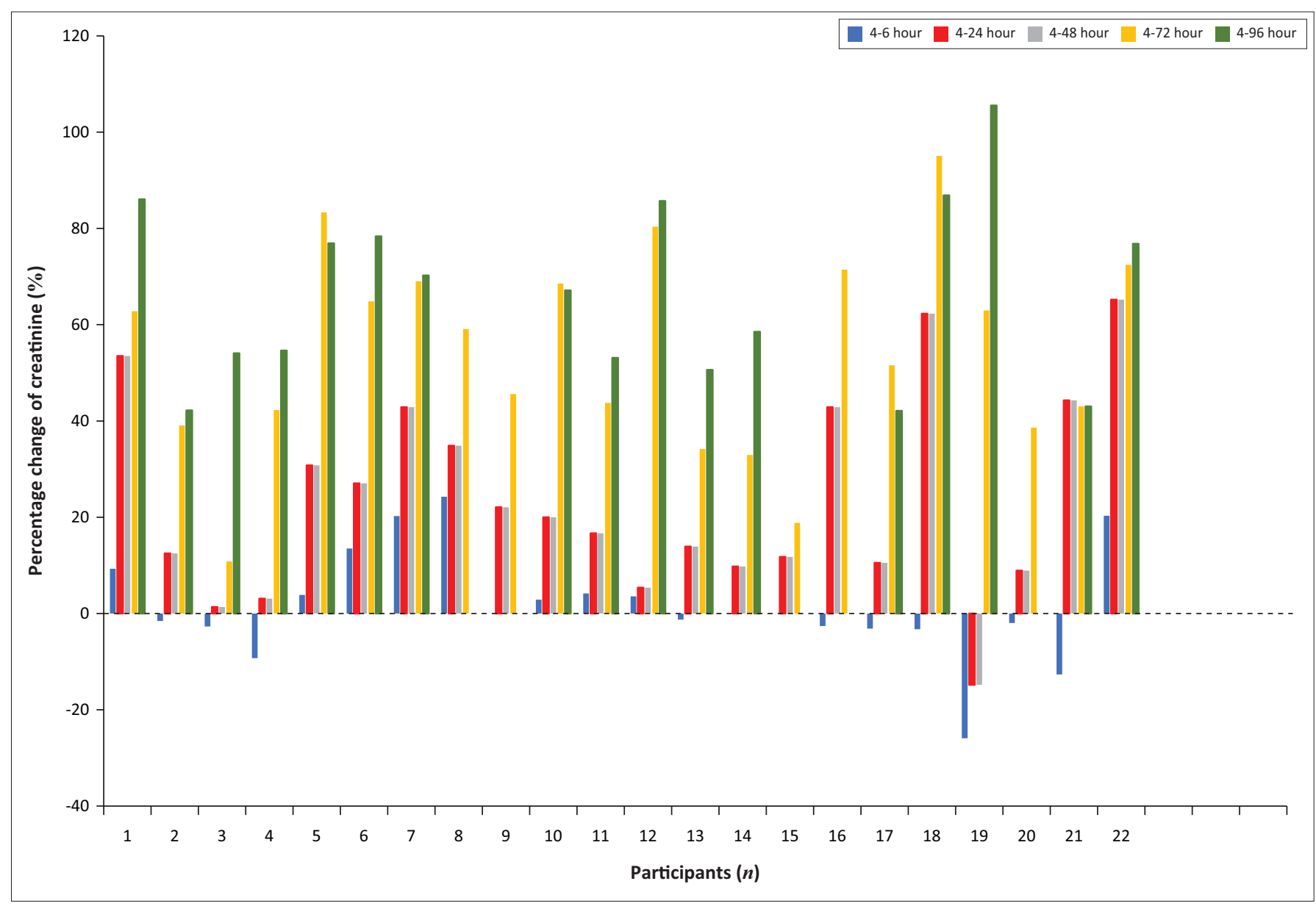

FIGURE 2: Percentage change in serum creatinine concentrations obtained using the kinetic Jaffe method over $96 \mathrm{~h}$ for 22 participants relative to the serum creatinine results at $4 \mathrm{~h}$. Percentage change to baseline $(4 \mathrm{~h})$ : blue bars $=4-6 \mathrm{~h}$, orange $=4-24 \mathrm{~h}$, grey $=4-48 \mathrm{~h}$, yellow $=4-72 \mathrm{~h}$, green $=4-96 \mathrm{~h}$.

\section{Method comparison}

Passing-Bablok regression and Bland-Altman plots were used to determine the correlation and agreement of the kinetic Jaffe method and i-STAT creatinine results compared to the enzymatic creatinine results at different time intervals (see Table 2, Figure 3 and Figure 4). The enzymatic method was chosen as the reference method as it had the best analytical CV in our study and previous studies have shown that this method correlated well with the standard reference method (isotopic dilution mass spectrometry). ${ }^{27,28,29}$

The Jaffe and enzymatic method showed a strong correlation at $<4 \mathrm{~h}(r=0.953)$; however, this correlation became weaker with time. The higher $r$ values at $72 \mathrm{~h}$ and $96 \mathrm{~h}$ may be due to the missing creatinine data (enzymatic $72 \mathrm{~h} n=3$, enzymatic $96 \mathrm{~h} n=6$, Jaffe $72 \mathrm{~h} n=1$, Jaffe $96 \mathrm{~h} n=4$ ) for participants at these time intervals. The slope and the intercept increased over the time interval (Table 2), and this demonstrated the increase in the magnitude of the systematic error. At $4 \mathrm{~h}$, there was a strong agreement between the kinetic Jaffe method and enzymatic methods with a small negative bias of $1.8 \%(1.7 \mu \mathrm{mol} / \mathrm{L})$. With an increased delay in sample separation, the kinetic Jaffe method resulted in an overestimation of creatinine concentrations with a positive bias of $48.6 \%(49.9 \mu \mathrm{mol} / \mathrm{l})$ at 96 h (Figure 3).
Creatinine results from the i-STAT correlated well with the enzymatic creatinine results over the six time intervals used in the study: $r$-value, $0.836-0.948$. The i-STAT method had a negative bias ranging from $7.4 \%$ to $18.0 \%$ throughout the study. The i-STAT method had a negative bias of $12.2 \%$ $(8.6 \mu \mathrm{mol} / \mathrm{L})$ at $4 \mathrm{~h}$ and $18.0 \%(12.0 \mu \mathrm{mol} / \mathrm{L})$ at $96 \mathrm{~h}$.

\section{Comparison of estimated glomerular filtration rate equation performance}

The impact on the classification of renal dysfunction of the delay in centrifugation of blood and serum samples measured for creatinine levels by means of three different laboratory methsods was evaluated with four eGFR equations viz. CG, MDRD v4, MDRD v3, and CKD-EPI. The serum creatinine eGFR results of the enzymatic and i-STAT methods performed well (to within the 10\% TAE for eGFR throughout the study). However, the eGFR from the Jaffe data decreased over time. At baseline, viz. $<4 \mathrm{~h}$, there was consensus among all four equations. All 22 participants had an eGFR $>60 \mathrm{~mL} / \mathrm{min}$ per $1.73 \mathrm{~m}^{2}$ using the CG and MDRD v4 equation. Similarly, 21 participants had an eGFR $>60 \mathrm{~mL} / \mathrm{min}$ per $1.73 \mathrm{~m}^{2}$ with the MDRD v3 and the CKD-EPI equations (Table 3). One participant had an eGFR of $<60 \mathrm{~mL} /$ min per $1.73 \mathrm{~m}^{2}$. This person was classified as having stage $3 \mathrm{~A}$ renal failure with an eGFR $45 \mathrm{~mL} / \mathrm{min}-59 \mathrm{~mL} / \mathrm{min}$ per $1.73 \mathrm{~m}^{2}$, according to the KDIGO guidelines. 


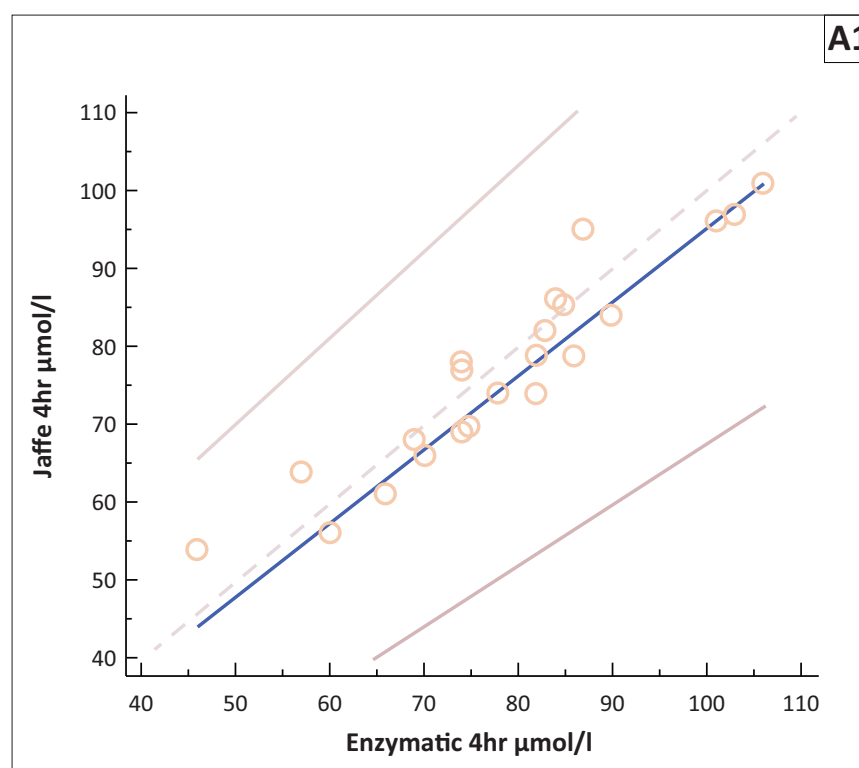

A1
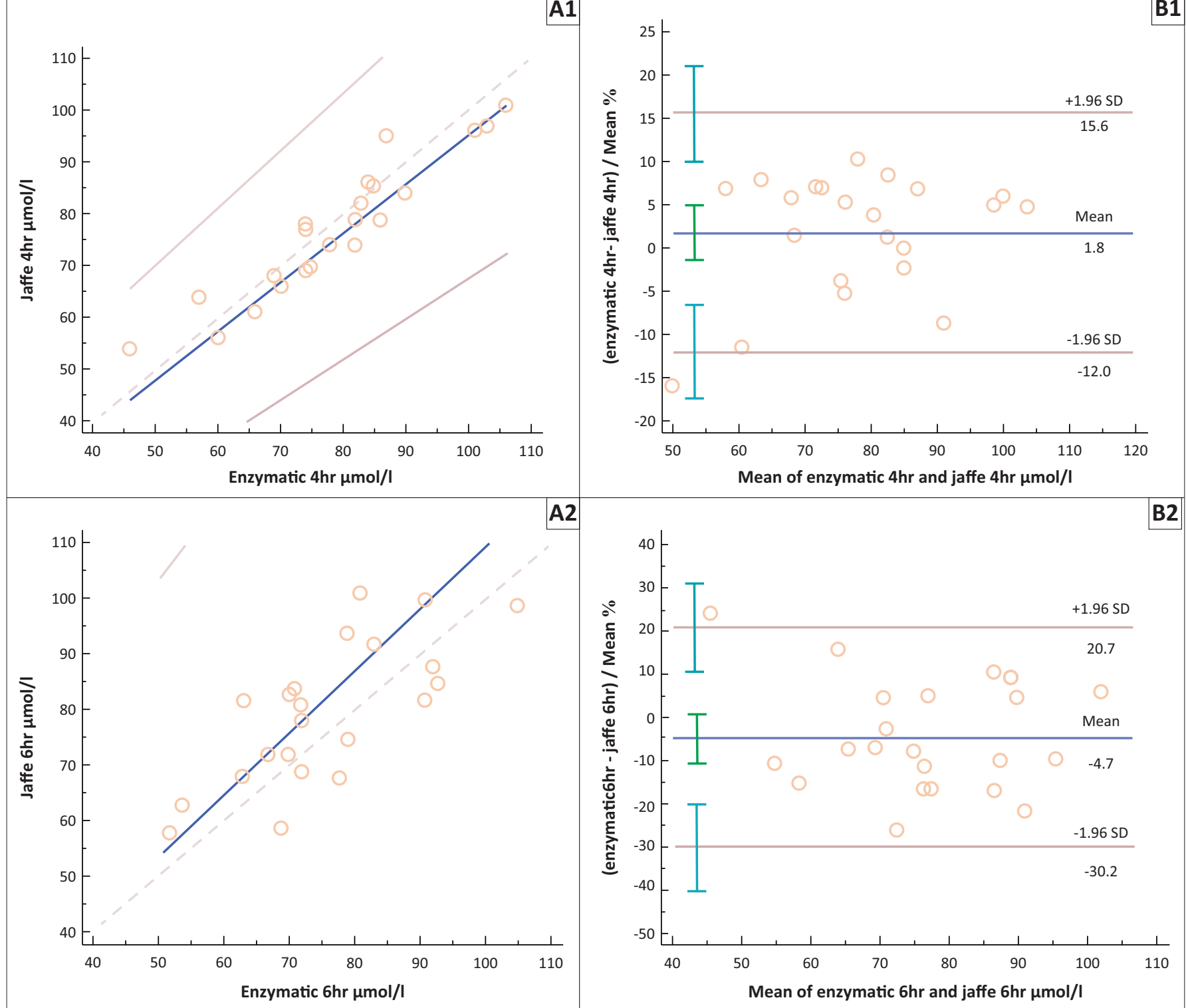

A3
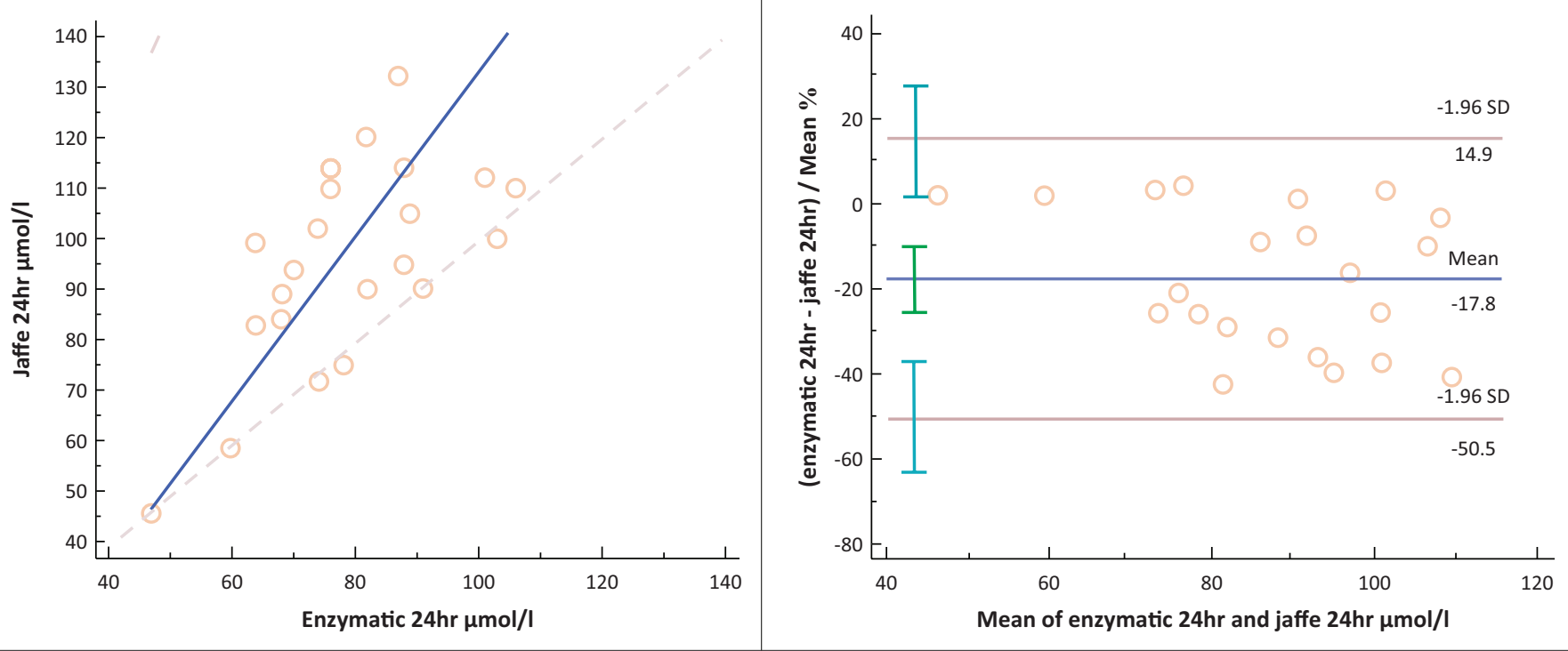

FIGURE 3: Passing-Bablok regression curves (A) and Bland-Altman plots (B) comparing creatinine concentrations from the kinetic Jaffe method with those from the enzymatic method. Creatinine concentrations at (1)< 4 h, (2) 6 h, (3) 24 h, (4) 48 h, (5) 72 h and (6) 96 h. 


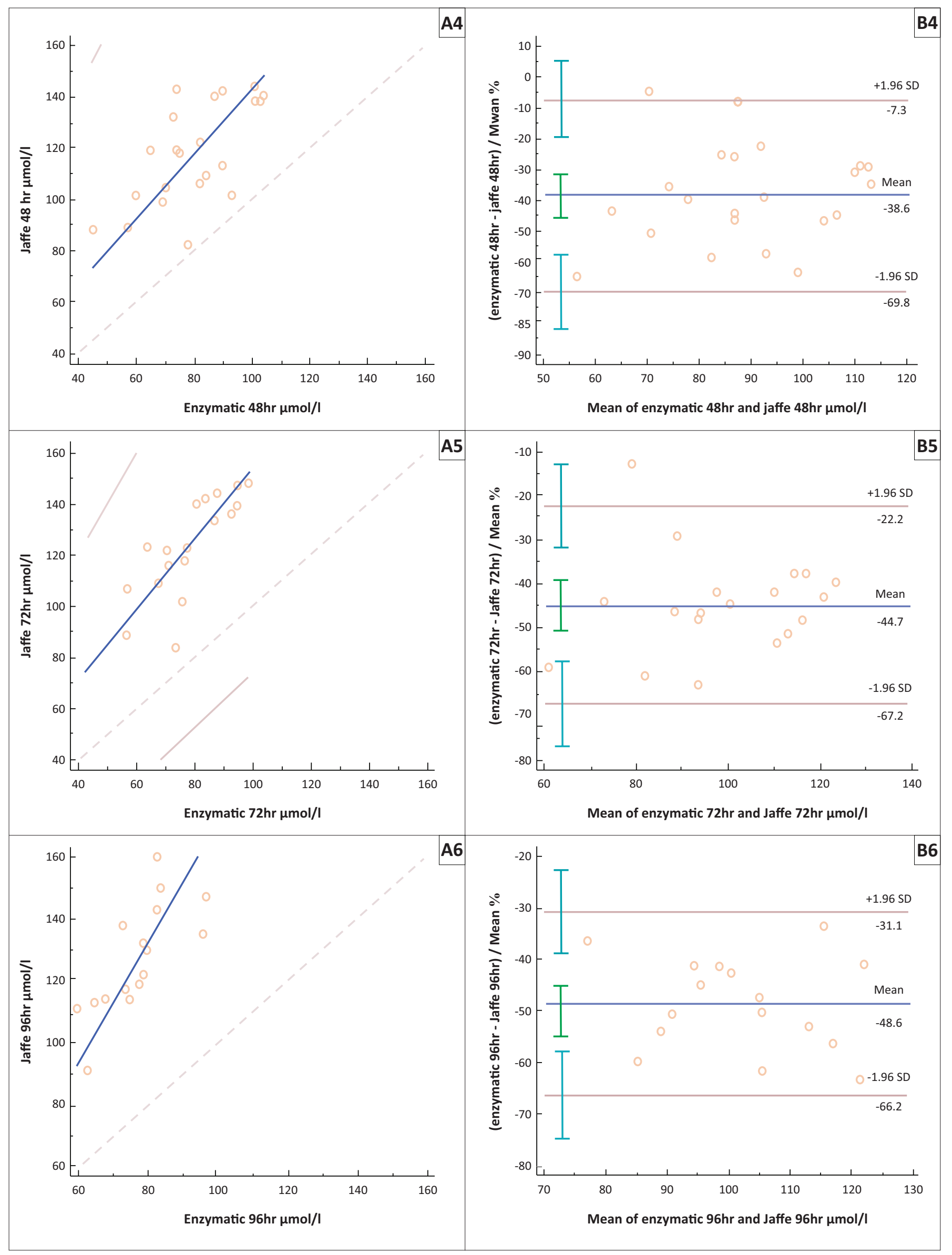

FIGURE 3 (Continues...): Passing-Bablok regression curves (A) and Bland-Altman plots (B) comparing creatinine concentrations from the kinetic Jaffe method with those from the enzymatic method. Creatinine concentrations at (1)<4 h, (2) $6 \mathrm{~h},(3) 24 \mathrm{~h},(4) 48 \mathrm{~h},(5) 72 \mathrm{~h}$ and (6) $96 \mathrm{~h}$. 

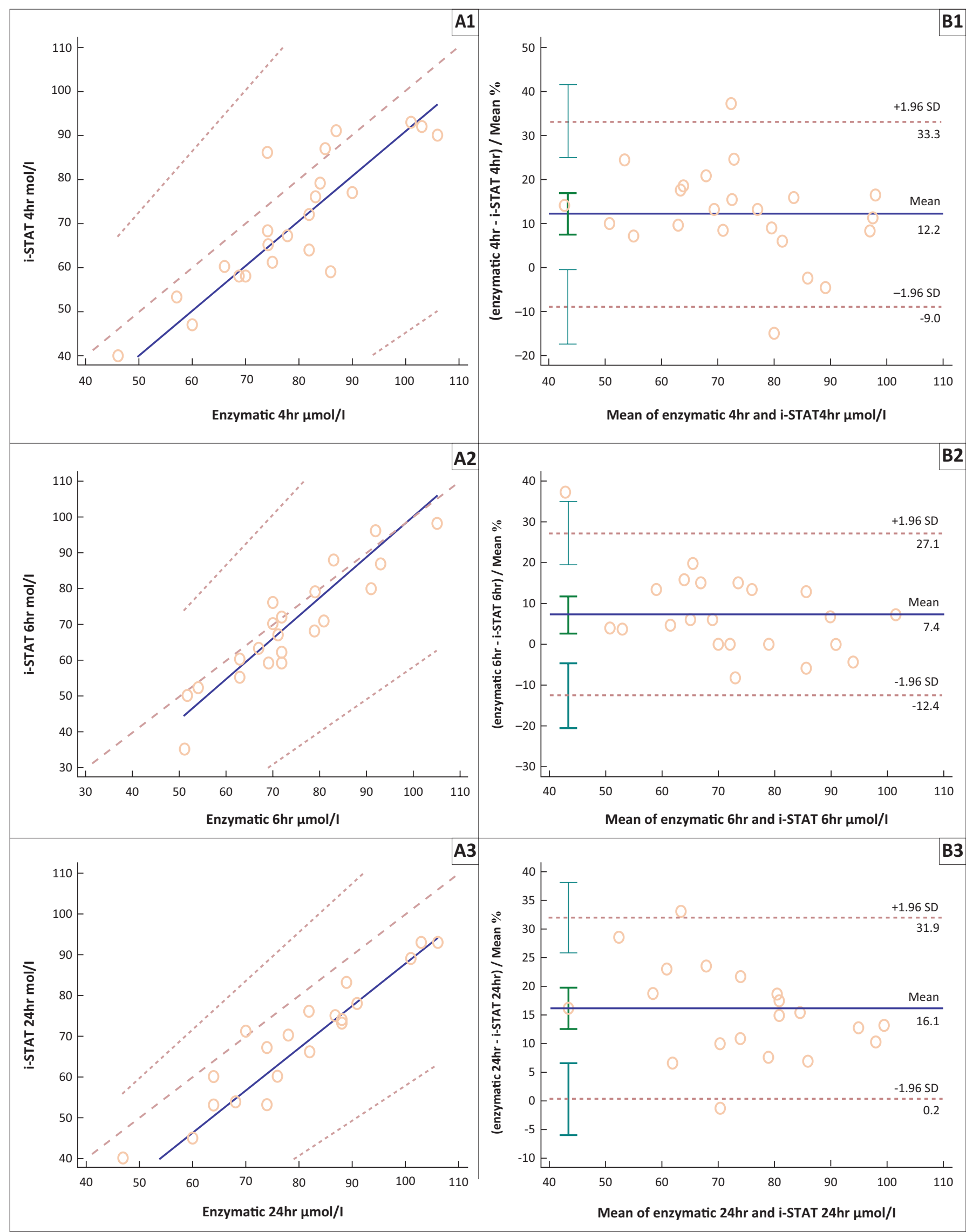

FIGURE 4: Passing-Bablok regression curves (A) and Bland-Altman plots (B) comparing creatinine concentrations from the i-STAT device with those obtained from the enzymatic method. Creatinine concentrations at (1) < 4 h, (2) 6 h, (3) 24 h, (4) 48 h, (5) 72 h and (6) 96 h. 


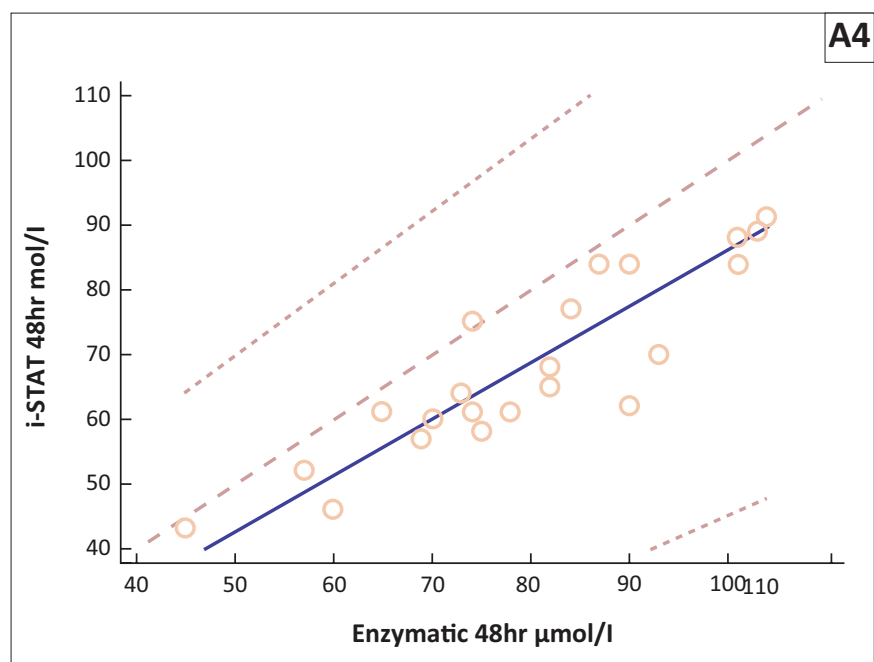

A4
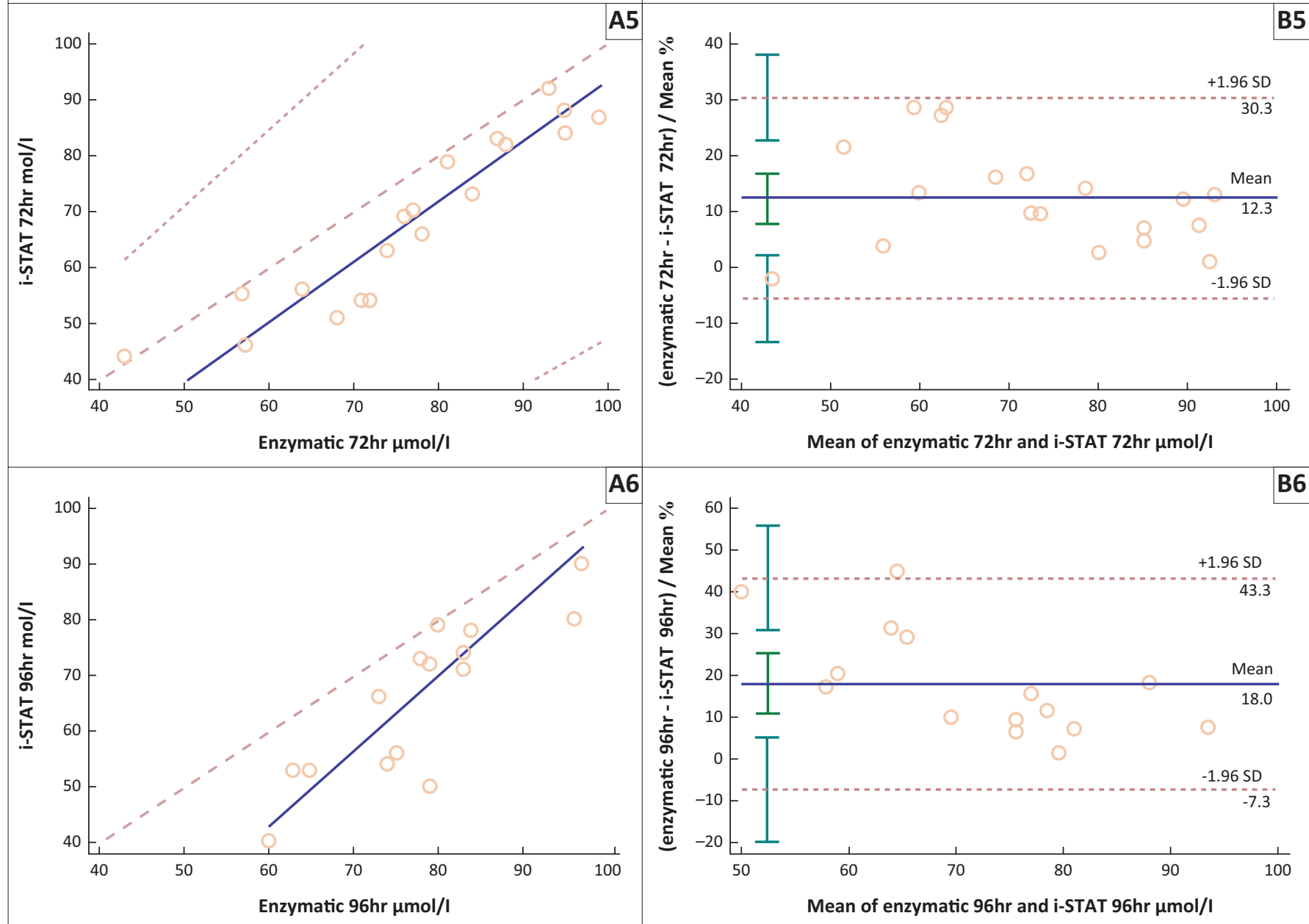

FIGURE 4 (Continues...): Passing-Bablok regression curves (A) and Bland-Altman plots (B) comparing creatinine concentrations from the i-STAT device with those obtained from the enzymatic method. Creatinine concentrations at (1)< $4 \mathrm{~h},(2) 6 \mathrm{~h},(3) 24 \mathrm{~h},(4) 48 \mathrm{~h},(5) 72 \mathrm{~h}$ and (6) $96 \mathrm{~h}$.

The change in eGFR varied over time among the different equations. The CG equation was the least sensitive to the increase in serum creatinine: only $27 \%(n=6 / 22)$ of eGFRbased $96-\mathrm{h}$ creatinine concentrations resulted in a change in the Chronic Kidney Disease (CKD) stage when compared with baseline (i.e. the < 4-h eGFR; see Figure 5). The MDRD v4 equation gave the greatest change in eGFR over $96 \mathrm{~h}: 50 \%$ $(n=11 / 22)$ of participants at $24 \mathrm{~h}$ and $100 \%(n=22 / 22)$ at $96 \mathrm{~h}$ showed an eGFR decline compared to baseline. The most significant change of eGFR was noted with the MDRDv3 equation: $25 \%$ eGFR at $48 \mathrm{~h}$ and $10 \%$ at $96 \mathrm{~h}$ compared to the baseline eGFR.

Using the CKD-EPI equation as recommended by the KDIGO guidelines, enzymatic and i-STAT renal classification was noted at different time intervals with changes between stages 1 and 2, which are not clinically significant for ARV drug choice. Using the kinetic Jaffe method however, changes in the renal classification were observed for 21 participants over the 96-h study period. 
TABLE 2: Comparison of serum creatinine levels from kinetic Jaffe method and i-STAT method to the enzymatic method using Passing-Bablok and Bland-Altman analysis.

\begin{tabular}{|c|c|c|c|c|c|c|c|c|c|}
\hline \multirow[t]{2}{*}{ Analytical method } & \multirow[t]{2}{*}{ Time (hours) } & \multicolumn{4}{|c|}{ Passing-Bablok regression parameters } & \multicolumn{4}{|c|}{ Bland-Altman } \\
\hline & & Slope & $95 \% \mathrm{Cl}$ & $\begin{array}{l}\text { Intercept } \\
(95 \% \mathrm{CI})\end{array}$ & $95 \% \mathrm{Cl}$ & $r$ & Mean bias & $\%$ & $\begin{array}{c}\text { Mean bias, } \\
\mu \mathrm{mol} / \mathrm{I}\end{array}$ \\
\hline \multirow[t]{4}{*}{ Kinetic Jaffe Method } & $<4 \mathrm{~h}$ & 0.95 & $0.78-1.1$ & 0.54 & $-10.74-15.54$ & 0.953 & 1.8 & $-1.31-4.93$ & 1.7 \\
\hline & $24 \mathrm{~h}$ & 1.6 & $0.95-2.50$ & -27.83 & $-88.50-19.65$ & 0.596 & -17.8 & $-25.20-10.40$ & 16.3 \\
\hline & $48 \mathrm{~h}$ & 1.26 & $0.83-2.23$ & 16.84 & $-56.85-52.75$ & 0.582 & -38.6 & $-45.66--31.51$ & 37.8 \\
\hline & $72 \mathrm{~h}$ & 1.33 & $1.06-2.00$ & 18.90 & $-32.00-40.13$ & $0.858 \dagger$ & -44.7 & $-50.24-39.18$ & 44.0 \\
\hline \multirow[t]{6}{*}{ i-STAT } & $<4 \mathrm{~h}$ & 1.01 & $0.83-1.38$ & -10.53 & $-38.23-3.50$ & 0.836 & 12.2 & $7.38-16.96$ & 8.6 \\
\hline & $6 \mathrm{~h}$ & 1.13 & $0.91-1.40$ & -13.24 & $-33.00-2.50$ & 0.900 & 7.4 & $2.89-11.84$ & 4.6 \\
\hline & $24 \mathrm{~h}$ & 1.04 & $0.86-1.20$ & -16.30 & $-28.50--0.59$ & 0.923 & 16.0 & $12.48-19.65$ & 11.7 \\
\hline & $48 \mathrm{~h}$ & 0.88 & $0.67-1.12$ & -1.35 & $-21.54-13.67$ & 0.893 & 15.8 & $11.69-19.95$ & 11.6 \\
\hline & $72 \mathrm{~h}$ & 1.08 & $0.86-1.37$ & -14.61 & $-38.41-2.57$ & $0.948 \S$ & 12.3 & $7.93-16.74$ & 8.6 \\
\hline & $96 \mathrm{~h}$ & 1.35 & $1.00-2.00$ & -38.02 & $-90.00-10.00$ & 0.8479 & 18.0 & $10.86-25.16$ & 12.0 \\
\hline
\end{tabular}

Legend: $r=$ correlation coefficient, linear regression line $y=m x+c, c=$ intercept (constant error), $m=$ slope (proportional error), number of participants results analysed.

$\dagger, n=19$.

$\ddagger n=16$.

$\S, n=19$

ฯ, $n=16$.

At baseline, $<4$ h, 21 of 22 (95\%) participants had an eGFR $>60 \mathrm{~mL} / \mathrm{min} / 1.73 \mathrm{~m}^{2}$ (renal stage 1 and 2) while at $96 \mathrm{~h}$ only 1 of $18(6 \%)$ participant was classified as stage 2 . The remaining 17 participants $(94 \%)$ were classified as stage 3a or higher $\left(\mathrm{eGFR}<60 \mathrm{~mL} / \mathrm{min} / 1.73 \mathrm{~m}^{2}\right.$ ). Figure 6 demonstrates that renal staging deteriorated over the study period, with all participants (100\%) eligible for the tenofovir based regimen with an eGFR $>50 \mathrm{~mL} / \mathrm{min} / 1.73 \mathrm{~m}^{2}$ at $4 \mathrm{~h}$ while only four participants (22\%) would have been eligible for this regimen at $96 \mathrm{~h}$.

\section{Discussion}

The accurate measurement of serum and whole-blood creatinine is essential to the determination of the eGFR. Laboratory methods have been standardised, but concern remains regarding the impact on results of nonspecific analytical biases. ${ }^{30,31}$ This study from a clinical laboratory based at a large public hospital in SA reports levels of serum creatinine as assessed by three standard methods of measurement, following a delay in sample separation and centrifugation. The clinical significance of the delay was evaluated by calculating the eGFR of each of the 22 patient's serum samples by means of the following four equations: the CG, MDRD v4, MDRD v3, and CKD-EPI. Serum and whole blood creatinine as assessed by the enzymatic and i-STAT methods were stable throughout the study. However, we found that a delay in centrifugation and sample separation of $>6 \mathrm{~h}$ resulted in significantly raised creatinine concentrations when using the kinetic Jaffe method. This led to the misclassification of patients when the eGFR was determined using all four formulas (equations), and was consistent with previous studies..$^{29,32}$

The Roche enzymatic creatinine results were stable over the duration of the study, with a CV of $6 \%$, which was consistent with a previous study which showed that the enzymatic method meets the analytical performance specifications as stipulated by the National Kidney Disease Education
Program (NKDEP) (USA). ${ }^{33,34}$ The i-STAT method showed a mean negative bias of $13.6 \mu \mathrm{mol} / \mathrm{L}$ compared to the enzymatic method. Studies comparing the performance of the i-STAT device to other platforms have been inconsistent. $^{35,36,37,38}$ One study showed that the i-STAT overestimated creatinine results by $3.88 \mu \mathrm{mol} / \mathrm{L}$ in comparison to the Roche enzymatic creatinine results. This overestimation occurred predominantly at higher creatinine concentrations. In contrast, a study conducted on oncology patients found that mean creatinine concentrations obtained on the i-STAT system were $42.4 \mu \mathrm{mol} /$ lower than those obtained using the core laboratory method. ${ }^{35}$ The negative bias may be attributed to whole blood creatinine negative interferences. ${ }^{39}$ POCT creatinine whole blood samples are affected by the matrix effect (such as haematocrit) and are prone to negative bias. ${ }^{40}$

Despite the negative bias seen with the i-STAT device, the creatinine results were sufficiently sensitive to detect the participant with renal dysfunction using the MDRD v3 and CKD-EPI equations. There are currently no POCT analytical goals for creatinine and no UOM was available as this was a new platform. However, the negative bias and the CV were within the acceptable 'Laboratory Working Group of the NKDEP' total error goal of less than $10 \%$ for the eGFR $(\mathrm{CV}<8 \%$ and an analytical bias relative to IDMS < 5\%). ${ }^{41}$ Previous studies have cautioned utilisation of POCT creatinine due to failure to detect renal dysfunction; however, in our study the i-STAT did not erroneously classify any of the participants. ${ }^{42}$ It is worth noting that the equations for eGFR were derived using serum samples, therefore utilisation of these equations with whole blood samples should still be validated using measured GFR.

While standardisation of creatinine methods has addressed calibration biases, method-specific interferences remain problematic. ${ }^{30}$ In the current study, the Roche kinetic Jaffe 


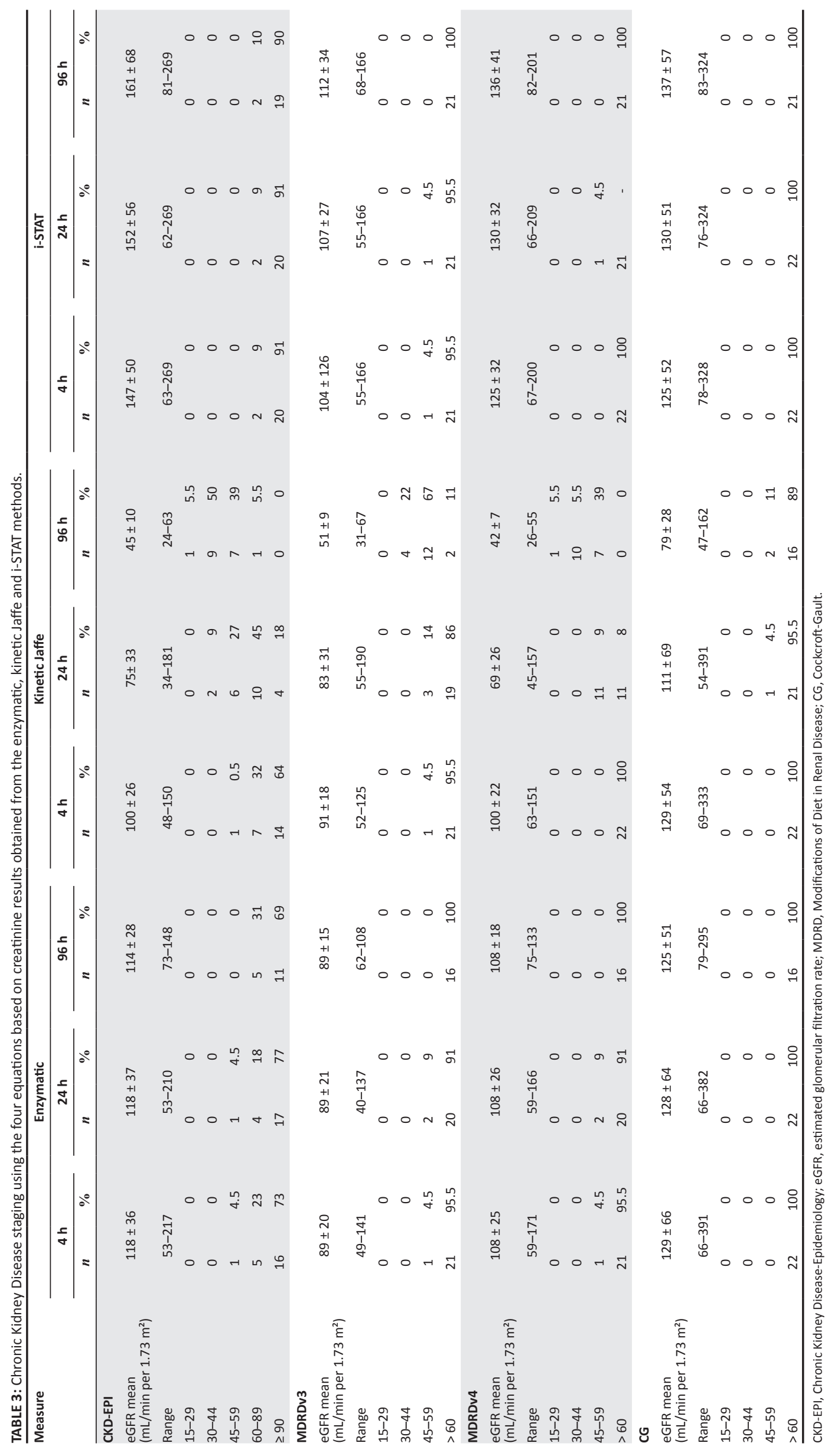




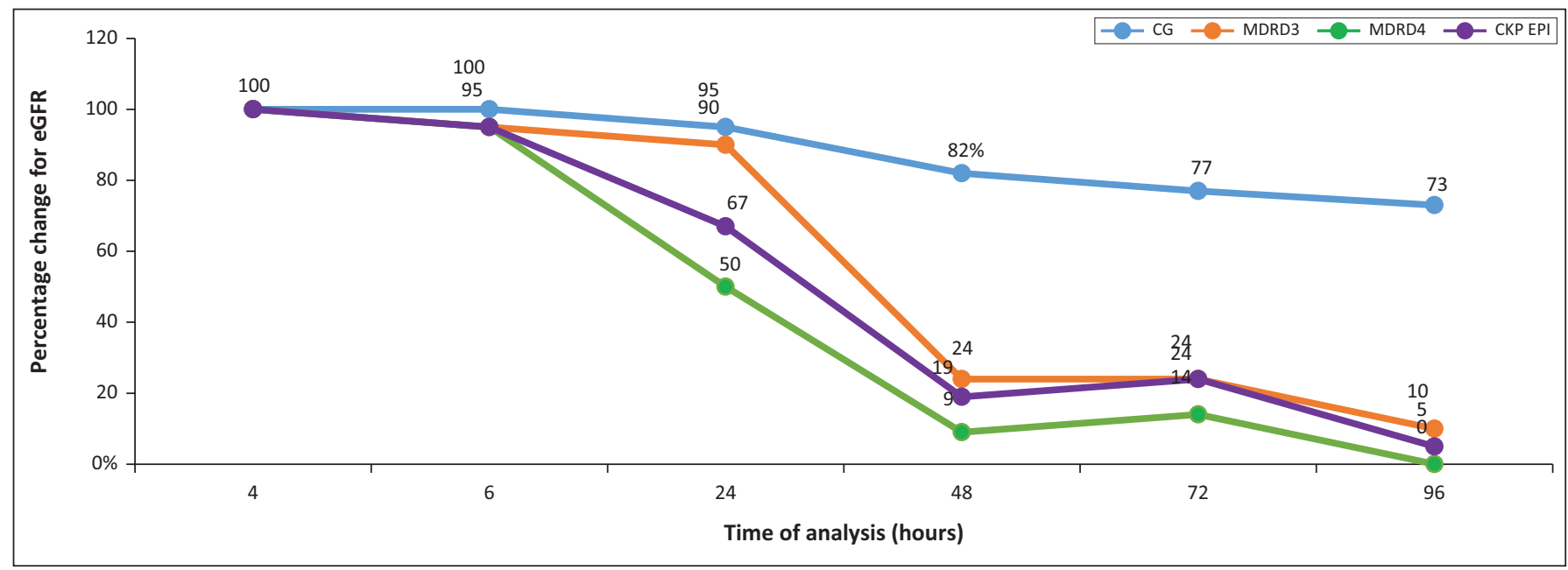

FIGURE 5: Percentage change of estimated glomerular filtration rate classification based on kinetic Jaffe creatinine concentrations over the $96-\mathrm{h}$ study period compared to estimated glomerular filtration rate obtained within $4 \mathrm{~h}$ using the Cockcroft-Gault, Modifications of Diet in Renal Disease v3, Modifications of Diet in Renal Disease v4, and Chronic Kidney Disease-Epidemiology equations. blue $=$ CG, orange $=$ MDRD3, purple = CKD, green = MDRD4.

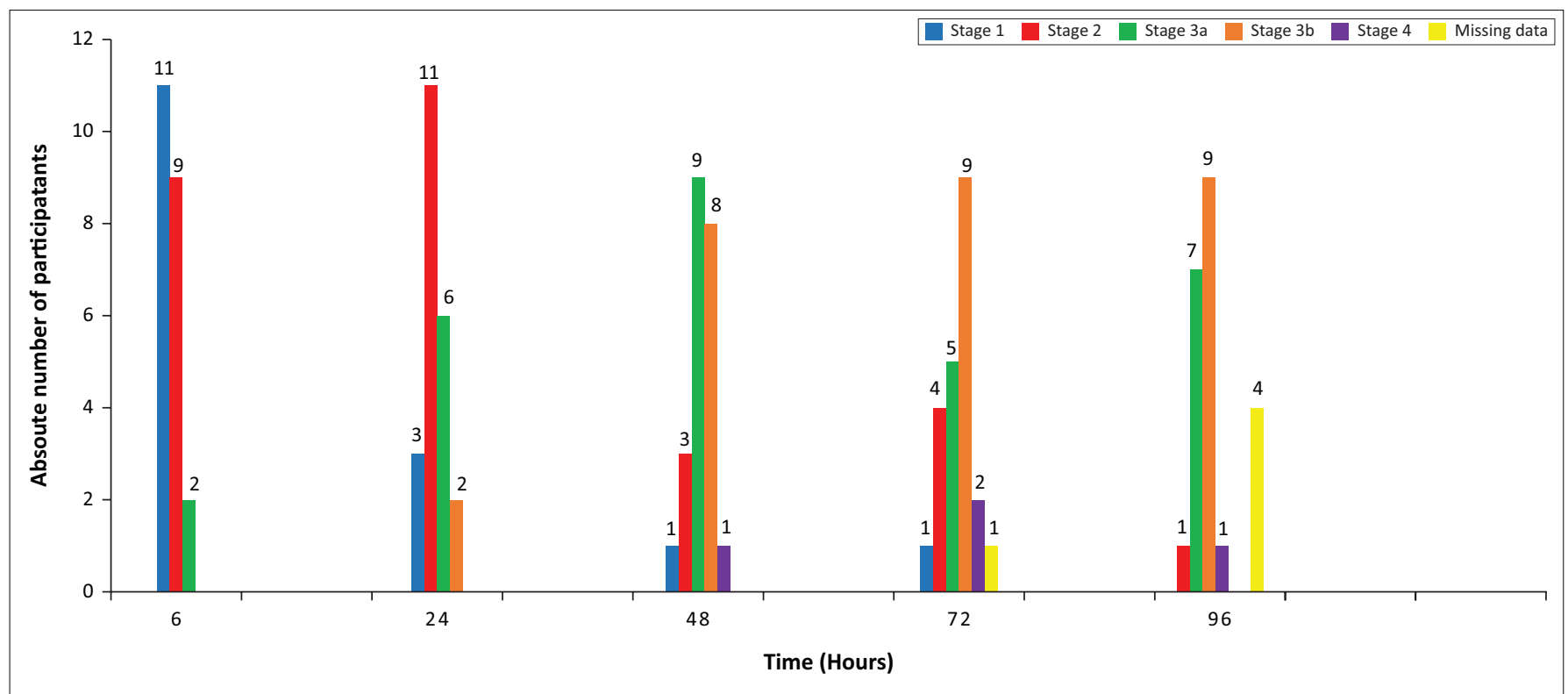

FIGURE 6: The number of participants classified per renal stage at each time interval using the serum creatinine results obtained from the kinetic Jaffe method to calculate estimated glomerular filtration rate using the Chronic Kidney Disease-Epidemiology equation. Blue bars $=$ stage 1 (eGFR $\geq 90 \mathrm{~mL} / \mathrm{min}$ per $1.73 \mathrm{~m}{ }^{2}$ ), Red bars $=$ stage 2 (eGFR $60 \mathrm{~mL} / \mathrm{min}$ per $1.73 \mathrm{~m}^{2}-89 \mathrm{~mL} / \mathrm{min}$ per $1.73 \mathrm{~m}^{2}$ ), Green bars = stage $3 a$ (eGFR $45 \mathrm{~mL} / \mathrm{min}$ per $1.73 \mathrm{~m}{ }^{2}-59 \mathrm{~mL} / \mathrm{min} \mathrm{per} 1.73 \mathrm{~m}{ }^{2}$ ), Orange bars $=$ stage $3 \mathrm{~b}(\mathrm{eGFR} 30$ $\mathrm{mL} / \mathrm{min}$ per $1.73 \mathrm{~m}^{2}-44 \mathrm{~mL} / \mathrm{min}$ per $\left.1.73 \mathrm{~m}^{2}\right)$, Purple bars = stage 4 (15 eGFR mL/min per $1.73 \mathrm{~m}^{2}-29 \mathrm{eGFR} \mathrm{mL} / \mathrm{min}$ per $1.73 \mathrm{~m}{ }^{2}$ ), Missing data due to haemolysis or insufficient sample $=$ yellow bars.

method showed analytical, clinically significant imprecision and a positive bias for creatinine results when analysed $24 \mathrm{~h}$ after collection. This finding is consistent with previous studies that showed delayed separation of serum from cellular components following specimen collection resulted in a positive interference with different Jaffe methods and could be attributed to the accumulation of pyruvate and other non-creatinine chromogens. ${ }^{26}$ Ford et al. ${ }^{32}$ established that on the Roche kinetic Jaffe method the serum creatinine results were significantly increased from $16 \mathrm{~h}$ and recommended that creatinine results with a delay in separation should not be reported as they impacted CKD staging. Similarly, Shepherd et al. ${ }^{26}$ showed an overestimation of Jaffe creatinine results when assessing the impact of delayed sample separation using five different analytical platforms. ${ }^{26}$ These results are in contrast to the WHO guidelines that state that creatinine in whole blood is stable at room temperature for $2-3$ days. ${ }^{25}$ In the current study, the overestimation by the kinetic Jaffe method over time was clinically significant as this led to a higher CKD staging (i.e. lower eGFR). This is consistent with a study by Drion et al. where they found that creatinine results from Jaffe techniques were more biased, imprecise and overestimated serum creatinine concentrations, especially at low concentrations compared to enzymatic techniques. ${ }^{29}$ The authors recommended that clinical laboratories consider the health costs associated with inappropriate referral and further suggested using the enzymatic assay that had a minimal bias.

The inconsistencies observed in renal classification using the four GFR equations shown in this study highlight the fact that these equations cannot be used interchangeably for 
identifying kidney disease, monitoring of renal function, drug dosing and selection of ARV drug regimens. Measured GFR was not required for this study as ascertaining the GFR was not pertinent, but rather the study sought to quantify the clinical significance of the impact of the instability on the calculated GFR. The CG equation was the least sensitive to the effect of the increased creatinine concentrations on eGFR in our study. This is consistent with a previous finding that demonstrated that the CG equation overestimated the eGFR in a large cohort of Europeans, resulting in the misclassification of $29.2 \%$ of individuals. ${ }^{43}$ In contrast, the CKD-EPI equation was sensitive to the serum creatinine changes of the kinetic Jaffe method, and the impact on the eGFR was noted at $24 \mathrm{~h}$. This result is consistent with a previous study by Seape and colleagues who found that the CKD-EPI equation performed better than other creatininebased eGFR equations when assessing renal function in 100 treatment-naïve HIV-positive individuals. ${ }^{44}$

\section{Strengths and limitations}

This study was able to show the impact of delayed processing of samples on creatinine concentrations on three platforms with a larger sample size than previous studies. In addition, blood samples were taken from participants at a single time point and the pre-analytical conditions were standardised. A limitation of this study is that serum indices (such as bilirubin which can falsely elevate creatinine results) were not considered when evaluating the creatinine result. Furthermore, we did not exclude all confounders to creatinine measurements such as drugs and pre-existing renal dysfunction which may have adversely affected the creatinine results at all time intervals. In addition, we did not use the reference creatinine method (IDMS) for comparison purposes; we used an enzymatic method traceable to IDMS. A further limitation is that we did not have a wide range of creatinine concentrations to see how a delay in centrifugation would affect the higher concentrations. Furthermore, this study did not explore all the risks associated with POCT devices such as lot-to-lot variation (one lot number was used), operator variability, quality control, analytical traceability, impact of temperature, humidity, different sample types, and the different analytical performance of different POCT devices which have previously been shown to have a significant impact on the eGFR. ${ }^{45}$

\section{Conclusion}

This study demonstrated that the Roche kinetic Jaffe method creatinine assay gave falsely elevated serum creatinine levels if samples were not separated and analysed within 24 $\mathrm{h}$ of collection. The increase in the serum creatinine concentration over time was clinically significant resulting in misclassification of renal status which would, in turn, lead to incorrect clinical decisions regarding ARV regimen choice. Based on the outputs of this study, and previously published literature, ${ }^{26,44}$ laboratories currently analysing creatinine on any Jaffe method should reject (not analyse) samples reaching the laboratory after the stability index time from collection $(<24 \mathrm{~h})$, or if these laboratories decide to analyse these serum creatinine samples an appropriate interpretative comment should be appended to the result as done for other biochemical analytes such as potassium, glucose, and phosphate. The enzymatic creatinine method is highly recommended in clinical laboratories that analyse samples more than $24 \mathrm{~h}$ after collection due to transport delays and excessive workload. A major deterrent concerning the utility of the enzymatic method is its high cost. However, the health costs associated with the misclassification of patients using the Jaffe method may outweigh the analytical costs of the enzymatic method. Increased demand for the enzymatic method will result in competition between suppliers and ultimately reduce the cost. This study demonstrated that although creatinine assays have been standardised the extra analytical considerations which are not standardised may result in clinically significant creatinine results, thus the use of POCT, which performed well in this study, in remote clinics may avert the need to perform the laboratory-based kinetic Jaffe.

Measurement of creatinine with a POCT provides an accurate, timely result, and has eliminated the pre-analytical problems noted with the kinetic Jaffe method; therefore, adopting of POCT creatinine should be envisaged. The i-STAT POCT creatinine, however, had a negative bias which resulted in the misdiagnosis of a renal dysfunction for one participant with stage 3a renal failure while the eGFR of the other 21 participants was not affected. Its utilisation in HIV/AIDS programmes could, therefore, be adopted once the eGFR equations have been validated for the sample type, confounders of POCT mentioned previously and infrastructural issues such as integration with the laboratory information systems have been addressed. POCT and enzymatic assays will reduce unnecessary clinic visits due to renal misdiagnoses (false positives) and importantly reduce the pill burden, thereby ultimately reducing health costs to both the government and the patient.

\section{Acknowledgements}

NHLS medical technologist team from Chris Hani Baragwanath Hospital, the Alexandra Health Clinic chronic care team, Mr Philani Buthelezi

\section{Competing interests}

The authors have declared that no competing interest exists.

\section{Authors' contributions}

C.E.C. recruited patients, collected data, obtained ethics approval and wrote the first draft. C.P. reviewed and edited the manuscript. D.T. conceptualised the study, reviewed the statistical analysis, reviewed and edited the manuscript and approved the final version of the manuscript. 


\section{Funding information}

We received the i-STAT machine and the cartridges from Obsidian Health (Pty) Ltd the official distributor of Abbott Point of Care South Africa. The company did not influence the protocol formulation, recruitment of participants, data analysis or the final write-up.

\section{Data availability statement}

Data from this study is available.

\section{Disclaimer}

The views and opinions expressed in this article are those of the authors and do not necessarily reflect the official policy or position of any affiliated agency of the authors.

\section{References}

1. Meintjes G, Moorhouse MA, Carmona S, et al. Adult antiretroviral therapy guidelines 2017. South Afr J HIV Med. 2017;18(1):1-24. https://doi.org/10.4102/ sajhivmed.v18i1.776

2. HIV Country Profiles. [cited 2019 Sep 17]. Available from: http://cfs.hivci.org/ country-factsheet.html

3. Kitahata MM, Gange SJ, Abraham AG, et al. Effect of early versus deferred antiretroviral therapy for HIV on survival. N EngI J Med. 2009;360(18):1815-1826. antiretroviral therapy for HIV on survival.
https://doi.org/10.1056/NEJMoa0807252

4. Jamieson DJ, King CC, Kourtis AP. Human immunodeficiency virus infection. Clin Gynecol. 2015;(2)360-370. https://doi.org/10.1017/CBO9781139628938.026

5. Capeau J. Premature aging and premature age-related comorbidities in HIVinfected patients: Facts and hypotheses. Clin Infect Dis. 2011;53(11):1127-1129. https://doi.org/10.1093/cid/cir628

6. Department of Health, the Republic of South Africa. The South African antiretroviral treatment guidelines, 2013 [homepage on the Internet]. [cited 2019 Aug 3] Available from: http://www.kznhealth.gov.za/medicine/2013_ART_Guidelines.pdf

7. Cotte L, Ferry T, Pugliese $P$, Valantin M-A. Effectiveness and tolerance of single tablet versus once daily multiple tablet regimens as first-line antiretroviral therapy - Results from a large french multicenter cohort study. PLoS One. 12(2):e0170661. https://doi.org/10.1371/journal.pone.0170661

8. Ghandi M, Ghandi RT. Single-pill combination regimens for treatment of HIV-1 infection. $N$ Engl J Med. 2014;371:248-259. https://doi.org/10.1056/ NEJMct1215532

9. Department of Health, Republic of South Africa. 2019 ART clinical guidelines for the management of HIV in adults, pregnancy, adolescents, children, infants and neonates [homepage on the Internet]. Dolutegravir Overview pg. $8 \mathrm{ff}$ 2019. Available from: https://sahivsoc.org/Files/2019\%20Abridged $\% 20$ ART $\% 20$ Guidelines\%2010\%200ctober\%202019.pdf

10. Venter WDF, Moorhouse M, Sokhela S, et al. Dolutegravir plus two different prodrugs of tenofovir to treat HIV. N Engl J Med. 2019;381(9):803-815. https:// doi.org/10.1056/NEJMoa1902824

11. Tourret J, Deray G, Isnard-Bagnis C. Tenofovir effect on the kidneys of hiv-infected patients: A double-edged sword? J Am Soc Nephrol. 2013;24(10):1519-1527. patients: A double-edged sword? J Am Soc
https://doi.org/10.1681/ASN.2012080857

12. Kohler JJ, Hosseini SH, Hoying-Brandt A, et al. Tenofovir renal toxicity targets mitochondria of renal proximal tubules. Lab Investig. 2009;89:513-519. https:// doi.org/10.1038/labinvest.2009.14

13. Wyatt CM, Klotman PE. Kidney disease in HIV infection: Introduction. Semin Nephrol. 2008;28(6):511-512. https://doi.org/10.1016/j.semnephrol.2008.08.004

14. Foy MC, Estrella MM, Lucas GM, et al. Article comparison of risk factors and outcomes in HIV immune complex kidney disease and HIV-associated nephropathy. Clin J Am Soc Nephrol. 2013;8(9):1524-1532. https://doi. org/10.2215/CJN.10991012

15. Alving AS, Miller BF. A practical method for the measurement of glomerular filtration rate (inulin clearance): With an evaluation of the clinical significance of this determination. Arch Intern Med. 1940;66(2):306-318. https://doi. org/10.1001/archinte.1940.00190140014002

16. Traynor J, Mactier R, Geddes CC, et al. Clinical review how to measure renal function in clinical practice. Br Med J. 2006;333(7571):733-737. https://doi org/10.1136/bmj.38975.390370.7C

17. Official Journal of the international society of nephrology KDIGO 2012. Clinical practice guideline for the evaluation and management of chronic kidney disease [homepage on the Internet]. [cited 2020 Mar 06] Available from: https://www. publicationethics.org

18. National Institute for Health and Care Excellence. 2014 clinical guideline. Chronic kidney disease in adults: Assessment and management [homepage on the Internet]. Available from: https://www.nice.org.uk/guidance/cg182
19. Bargnoux A-S, Kuster N, Cavalier E, et al. Serum creatinine: advantages and pitfalls. J Lab Precis Med. 2018;3(8):71.

20. Naicker J. Glomerular filtration rate (GFR) and estimation of the GFR (eGFR) in adults. Continuing Med Educ [serial online]. 2012;30(7). Available from: https:// www.cmej.org.za/index.php/cmej/article/view/2514/2430

21. Cockcroft DW, Gault MH. Prediction of creatinine clearance from serum creatinine. Nephron. 1976;16(1):31-41. https://doi.org/10.1159/000180580

22. Levey AS, Bosch JP, Lewis JB, et al. A more accurate method to estimate glomerular filtration rate from serum creatinine: A new prediction equation. Ann Intern Med. 1999;130(6):461-470. https://doi.org/10.7326/0003-4819130-6-199903160-00002

23. Van Deventer HE, George JA, Paiker JE, et al. Estimating glomerular filtration rate in black South Africans by use of the modification of diet in renal disease and Cockcroft-Gault equations. Clin Chem. 2008;54(7):1197-202. https://doi. org/10.1373/clinchem.2007.099085

24. Levey AS, Stevens LA, Schmid CH, et al. A new equation to estimate glomerular filtration rate. Ann Intern Med. 2009;150(9):604-612. https://doi.org/10 7326/0003-4819-150-9-200905050-00006

25. World Health Organization. Diagnostic Imaging and Laboratory Technology. [Use of anticoagulants in diagnostic laboratory investigations [homepage on the Internet] World Health Organization; 2002. Available from: https://apps.who.int/iris/ handle/10665/65957

26. Shepherd J, Warner MH, Kilpatrick ES. Stability of creatinine with delayed separation of whole blood and implications for eGFR. Ann Clin Biochem. 2007;44(4):384-387. https://doi.org/10.1258/000456307780945660

27. Gómez-Rioja R, Segovia Amaro M, Diaz-Garzón J, et al. Guidelines and recommendations from scientific societies a protocol for testing the stability of biochemical analytes. Technical document. Clin Chem Lab Med. 2019;57(12) 1829-1836. https://doi.org/10.1515/cclm-2019-0586

28. Badiou S, Dupuy AM, Descomps B, Cristol JP. Comparison between the enzymatic vitros assay for creatinine determination and three other methods adapted on the olympus analyzer. J Clin Lab Anal. 2003;17(6):235-240. https://doi.org/10.1002/ jcla.10103

29. Drion I, Cobbaert C, Groenier KH, et al. Clinical evaluation of analytical variations in serum creatinine measurements: Why laboratories should abandon Jaffe techniques. BMC Nephrol. 2012;13(1):1-8. https://doi.org/10.1186/1471-236913-133

30. Jassam N, Weykamp C, Thomas A, et al. Post-standardization of routine creatinine assays: Are they suitable for clinical applications. Ann Clin Biochem. 2017;54(3):386-394. https://doi.org/10.1177\%2F0004563216664541

31. Greenberg N, Roberts WL, Bachmann LM, et al. Specificity characteristics of 7 commercial creatinine measurement procedures by enzymatic and jaffe method principles. Clin Chem. 2012;58(2):391-401. https://doi.org/10.1373/clinchem. 2011.172288

32. Ford L, Berg J. Delay in separating blood samples affects creatinine measurement using the Roche kinetic Jaffe method. Ann Clin Biochem. 2008;45(1):83-87. https://doi.org/10.1258\%2Facb.2007.007109

33. Boutten A, Bargnoux AS, Carlier MC, et al. Enzymatic but not compensated Jaffe methods reach the desirable specifications of NKDEP at normal levels of creatinine. Results of the French multicentric evaluation. Clin Chim Acta. creatinine. Results of the French multicentric evaluation.
2013;419:132-135. https://doi.org/10.1016/j.cca.2013.01.021

34. Küme T, Sağlam B, Ergon C, Sisman AR. Evaluation and comparison of Abbott Jaffe and enzymatic creatinine methods: Could the old method meet the new requirements? J Clin Lab Anal. 2018;32(1):1-8. https://doi.org/10.1002/ jcla.22168

35. Bahar B, DeChristopher PJ, Holmes EW, Kahn SE Investigation of falsely decreased creatinine results observed from the abbott i-Stat point-of-care device in use for testing specimens from ambulatory oncology patients. Point device in use for testing specimens from ambulatory oncology patients. Point
Care J Near-Patient Test Technol. 2016;15(2):72-77. https://doi.org/10.1097/ Care J Near-Patient Test

36. Korpi-Steiner NL, Williamson EE, Karon BS. Comparison of three whole blood creatinine methods for estimation of glomerular filtration rate before radiographic creatinine methods for estimation of glomerular filtration rate before radiographic contrast administration. Am J Clin
org/10.1309/AJCPTE5FEYOVCGOZ

37. Snaith B, Harris MA, Shinkins B, Jordaan M, Messenger M, Lewington A. Point-of care creatinine testing for kidney function measurement prior to contrastenhanced diagnostic imaging: Evaluation of the performance of three systems for enhanced diagnostic imaging: Evaluation of the performance of three systems for
clinical utility. Clin Chem Lab Med. 2018;56(8):1269-1276. https://doi. clinical utility. Clin Chem
org/10.1515/cclm-2018-0128

38. van der Heijden $C$, Roosens $L$, Cluckers $H$, Van Craenenbroeck $A H$, Peeters $B$. Analytical and clinical performance of three hand-held point-of-care creatinine
analyzers for renal function measurements prior to contrast-enhanced analyzers for renal function measurements prior to contrast-enhanced
imaging. Clin Chim Acta. 2019;497(March):13-19. https://doi.org/10.1016/j. cca.2019.06.025

39. Straseski JA, Lyon ME, Clarke W, DuBois JA, Phelan LA, Lyon AW. Investigating interferences of a whole-blood point-of-care creatinine analyzer: Comparison to plasma enzymatic and definitive creatinine methods in an acute-care setting. Clin Chem. 2011;57(11):1566-1573. https://doi.org/10.1373/clinchem.2011. 165480

40. Shephard MDS. Point-of-care testing and creatinine measurement. Clin Biochem Rev. 2011;32(2):109-114.

41. Myers GL. Recommendations for improving serum creatinine measurement: A report from the laboratory working group of the national kidney disease education program. Clin Chem. 2006;52(1):5-18. https://doi.org/10.1373/clinchem.2005. 0525144 
42. Moreno M, Schwartz A, Dvorkin R. The accuracy of point-of-care creatinine testing in the emergency department. Adv Emerg Med. 2015;965368:5. https://doi. in the emergency departmer
$\mathrm{org} / 10.1155 / 2015 / 965368$

43. Froissart M, Rossert J, Jacquot C, Paillard M, Houillier P. Predictive performance of the modification of diet in renal disease and Cockcroft-Gault equations for estimating renal function. J Am Soc Nephrol. 2005;16(3):763-773. https://doi. org/10.1681/ASN.2004070549
44. Seape T, Gounden V, van Deventer HE, Candy GP, George JA. Cystatin $\mathrm{C}$ - and creatinine-based equations in the assessment of renal function in th-positive patients prior to commencing 63215579695 63215579695

45. Nichols JH, Nichols JH. Risk management for point-of-care testing.2014; 25(2):154-161. 